\title{
Trauma care at King Abdulaziz University hospital: A retrospective cohort study
}

\author{
Almarhabi Yahya ${ }^{1 *}$, AlQulayti W' ${ }^{2}$, Almaghrabi $S^{3}$, Tallab $\mathbf{M}^{4}$, Mukhtar Abdel Moniem ${ }^{5}$, Al-Abdulah Nabeela ${ }^{6}$ \\ ${ }^{\mathbf{1}}$ Assistant Professor, ${ }^{2-4}$ Medical Students, $5,{ }^{6}$ Associate Professor, ${ }^{1}$ Dept. of Surgery, ${ }^{5}$ Dept. of Family and Community Medicine, ${ }^{6}$ Dept. of \\ Public Health, ${ }^{1}$ Center of Excellence in Trauma and Accidents, Faculty of Medicine, King Abdulaziz University, Jeddah, Saudi Arabia, \\ ${ }^{2-5}$ Faculty of Medicine, King Abdulaziz University, Jeddah, Saudi Arabia, ${ }^{6}$ Faculty of Nursing, King Abdulaziz University, Jeddah, Saudi \\ Arabia
}

*Corresponding Author: Almarhabi Yahya

Email: yalmarhabiy@kau.edu.sa

\begin{abstract}
Introduction: In Saudi Arabia, road traffic accidents (RTAs) cause about 20 deaths per day and are the leading cause of death among young Saudi men. The aim of our study was to describe the diagnostic and therapeutic care provided to trauma patients admitted to the emergency department of the King Abdulaziz University Hospital (KAUH) in Jeddah, Saudi Arabia.

Materials and Methods: This is a descriptive, retrospective, hospital record-based study that includes all patients admitted to the emergency department at KAUH between January 2010 and January 2015 due to trauma. We extracted data on demographic characteristics, trauma type, mode of transportation, length of stay in the emergency department, clinical and laboratory investigations, imaging techniques, ward and intensive care unit admission, duration of hospital stay, status at discharge, and type and number of departments involved in patient care.

Results: Between 2010 and 2015, total 111 trauma patients were assessed in the emergency department(ER); their mean age was $26.54 \pm$ 18.09 years, $79.3 \%(n=88)$ were males, and $56.8 \%(n=63)$ were non-natives. Blood tests and radiological imaging were conducted in 106 $(95.5 \%)$ and $97(87.4 \%)$ patients, respectively. Subsequently, $96(86.5 \%)$ patients were admitted to various wards, $9(8.1 \%)$ were discharged, and $5(4.5 \%)$ died ( 2 in the ER, 3 in the ICU). Further, 34 (30.6\%) patients were eventually admitted to the ICU. The mean duration of hospital stay was $11.15 \pm 16.01$ days, and the mode of transport to the hospital was not documented for $69(62.2 \%)$ patients.

Conclusion: Trauma causalities pose a significant threat to population health and may drain healthcare resources. Prevention strategies are warranted to reduce injuries, deaths, and disabilities. Currently, trauma care at and accessibility to, the KAUH is suboptimal for trauma victims.
\end{abstract}

Keywords: Trauma system assessment, Acute Surgery Care, Road traffic accident.

\section{Introduction}

Trauma is one of the major concerns in surgical care. Unexpected injuries can quickly change lives with lasting consequences. Trauma is an international epidemic with an annual mortality rate of more than 5 million people worldwide; this translates to 1 death every 6 seconds due to accidents. ${ }^{1-3}$ Trauma can lead to poverty because of the direct and indirect costs of care. Reports suggest that over 5.1 million years and $\$ 150$ billion are lost annually due to injuries in the United States alone..$^{2-4}$ Importantly, the burden of trauma is not limited to physical and economic outcomes, as it also has an emotional and social impact on patients and their families.

In Saudi Arabia, road traffic accidents (RTAs) are associated with 20 deaths per day and are the leading cause of death among young Saudi men. Almost half a million RTAs were registered in Saudi Arabia between 2010 and 2011, which resulted in 7,159 fatalities and more than 40,000 injuries. Further, if no counter measures are taken, Saudi Arabia could have more than 4 million RTAs per year by $2030 .{ }^{5}$ Compared to other countries, RTAs in Saudi Arabia have a higher fatality rate at 29 deaths per $100,000{ }^{6}$ Annually, about $4 \%$ of the national income is lost due RTAs. ${ }^{7}$

There is limited research on trauma burden in Saudi Arabia. Consequently, a lack of understanding of the extent of this burden is manifested by limited investment in prevention strategies. The few studies that have described the burden were from the Central region, Riyadh in particular. The aim of this study was to describe various aspects of care provided to trauma patients admitted to the emergency department (ER) of King Abdulaziz University Hospital (KAUH), Jeddah, Saudi Arabia.

\section{Materials and Methods Study design and setting}

A retrospective cohort study was conducted in patients presenting to the ER of the KAUH, Jeddah, Saudi Arabia. KAUH is one of the major tertiary care and teaching centers in the western region of Saudi Arabia with a capacity of 800 beds. Jeddah is a coastal city on the Red Sea with a population of approximately $3,431,000$ individuals.

\section{Study population and study sample}

Based on prior data from Saudi Arabia, ${ }^{8}$ we expected a 0.5day standard deviation in the length of stay at the emergency department. Further, we would need to study 100 patients to estimate the length of hospital stay with a precision of 0.1 . Therefore, we included all patients $(\mathrm{n}=111)$ admitted to the emergency department at the KAUH between January 2010 and January 2015 due to trauma.

\section{Study tool}

Medical records of the KAUH were screened to identify all relevant patient records and data on the following variables were extracted. These include demographic characteristics, 
type of trauma, mode of transportation to the hospital, length of stay in the ER, clinical and laboratory investigations, imaging assessments, ward admission or intensive care unit admission, length of total hospital stay, status at end of hospitalization, and the type and number of departments involved in patient care. To reduce selection bias, we included all trauma patients and conducted plausibility checks and cross-validations to minimize measurement bias.

The study protocol was reviewed and approved by the Health Research and Ethics Board at King Abdulaziz University Hospital and the Biomedical Research Ethics Unit at King Abdulaziz University. (HA-02-J-008) and Registration number at the National Committee of Bio \& Medical Ethics (Reference No 64-16) and also funded by the Deanship of Scientific Research (DSR) at King Abdulaziz University, Jeddah, under grant no. J-742-14038. The authors therefore, acknowledge with thanks the DSR for technical and financial support. The DSR has no role in the design of the study, the collection, analysis and interpretation of data or in writing the manuscript.

\section{Statistical analysis}

Descriptive statistics such as counts, means, standard deviations, medians, and inter-quartile ranges (IQR) for continuous variables were used to describe the study population. To investigate the differences among patients according to their length of total hospital stay, we dichotomized the latter variable using its median value. To investigate the difference among patients based on trauma type, we dichotomized data as RTA and non-RTA. Between group differences in two categorical variables were assessed by using the Chi-square test or the Fisher exact test, if data were sparse. Between-group differences in continuous variables were estimated using the Student's t-test (unpaired, two samples) or one-way Analysis of Variance (ANOVA) when there were more than two groups. For all statistical tests, p-value of $<0.05$ was used to define significance. The Statistical Package for Social Sciences (SPSS) software (version 21) was used for all data analysis.

\section{Results}

It was seen from Table 1 that the electronic information system of the KAUHidentified111 trauma patients treated at the emergency department (ER) during the study period. The mean and median age of these patients were 26.54 years $(\mathrm{SD}=18.09$ years $)$ and $26(\mathrm{IQR}=27)$ respectively. Of these, $79.3 \%(\mathrm{n}=88)$ were males and $56.8 \%(\mathrm{n}=63)$ were nonnatives. The mean and median of the duration of initial assessment at the ER were 9.37 hours ( $\mathrm{SD}=10.37$ hours) and 5 hours (IQR= 10 hours), respectively. The mean and median of the duration of length of total stay in the hospital were 11.15 days $(\mathrm{SD}=16.01$ days) and 6 days $(\mathrm{IQR}=8$ days), respectively. In $62 \%(n=69)$ of the trauma patients, the mode of transport to the hospital had not been documented; however, records showed that $30.6 \%(n=34)$ of the patients arrived at the hospital by private vehicle and only $7.2 \%(\mathrm{n}=8)$ by ambulance. Further, $81 \%(\mathrm{n}=34)$ of the cases with a known pre-admission history arrived in private cars and only $19 \%(\mathrm{n}=8)$ by ambulance. Etiologically, $33.3 \%(n=37)$ of the cases were RTAs, $26.1 \%(n=29)$ were fall injuries, and $19.8 \%(n=22)$ were stab wounds. In RTAs, $86.5 \%(\mathrm{n}=32)$ of the cases had traumatic injuries to drivers and $13.5 \%(n=5)$ had affected pedestrians. Additionally, burns, drowning, and gun shots accounted for $7.2 \%(\mathrm{n}=8)$, $4.5 \%(\mathrm{n}=5)$ and $1.8 \%(\mathrm{n}=2)$ of the patients, respectively.

Blood tests $(95.5 \% ; \mathrm{n}=106)$ and radiological assessments $(87.4 \% ; \mathrm{n}=97)$ were conducted for most of the patients in the ER. Subsequently, $86.5 \%(n=96)$ of the patients were admitted to various wards, $30.6 \%$ (34) of the patients were eventually admitted to the ICU, $8.1 \%(n=9)$ were discharged, and $4.5 \%(n=5)$ died. Of these, 3 died in the first week after ICU admission and 2 died during emergency resuscitation within 2 hours after arrival. Vitals such as heart rate $(32.4 \% ; n=36)$, blood pressure $(30.6 \%$, $\mathrm{n}=34)$, oxygen saturation level $(30.6 \% ; \mathrm{n}=34)$, and body temperature $(27.9 \% ; n=31)$ were measured at arrival in only about one-third of the patients. A total of 135 consultations were requested for 82 patients $(73.87 \%)$ and were documented by subspecialty. The mean number of specialties involved in patient care (all subjects) was 1.67 $(\mathrm{SD}=0.08)$. Personnel from surgical departments, orthopedic departments, and both surgical and orthopedic departments were involved in patient care for $42.3 \%(\mathrm{n}=$ $47), 2.7 \%(n=3)$ and $7.2 \%(n=8)$ of the patients, respectively, while personnel from one, two, three, and four departments, were involved, in the care of $45.0 \%(n=50)$, $14.4 \%(n=16), 8.1 \%(n=9)$ and $6.3 \%(n=7)$ of the patients, respectively.

It was seen from Table 2 that patients were grouped based on duration of hospital stay as short ( $<6$ days) or long (> 6 days). Compared to trauma patients with a short hospital stay, long-stay patients had a significantly higher likelihood of admission to the ICU $(p<0.001)$, were more likely to be transported to the hospital by ambulance $(\mathrm{p}=0.02)$, required referrals and personnel from more departments $(\mathrm{p}=0.03)$, and showed a tendency to differ by type of trauma $(\mathrm{p}=0.08)$.

It was seen from Table 3 that no significant difference in length of stay as a function of age or other variables was found. Compared to trauma caused by RTAs, non-RTA trauma was significantly more common among non-natives $(\mathrm{p}=0.001)$, was treated by a smaller number of departments $(\mathrm{p}=0.04)$, and tended to undergo radiological assessment more frequently $(p=0.08)$. No significant difference in type of trauma was found when patients were grouped based on age or other variables. 
Table 1: Characteristics of trauma patients admitted to king abdulaziz university hospital $(\mathrm{n}=111)$

\begin{tabular}{|c|c|}
\hline Variable & Results \\
\hline $\begin{array}{l}\text { Age in Years } \\
\text { Mean (SD*) } \\
\text { Median }\left(\mathrm{IQR}^{* *}\right) \\
\text { Minimum - Maximum } \\
\text { Not Reported \% (n) }\end{array}$ & $\begin{array}{l}26.54(18.09) \\
26(27) \\
1-75 \\
0.0(0)\end{array}$ \\
\hline $\begin{array}{l}\text { Gender \% (n) } \\
\text { Male } \\
\text { Female } \\
\text { Not Reported }\end{array}$ & $\begin{array}{c}79.3(88) \\
19.8(22) \\
0.9(1)\end{array}$ \\
\hline $\begin{array}{l}\text { Nationality } \%(\mathrm{n}) \\
\text { Saudi } \\
\text { Non-Saudi } \\
\text { Not Reported }\end{array}$ & $\begin{array}{c}40.5(45) \\
56.8(63) \\
2.7(3)\end{array}$ \\
\hline $\begin{array}{l}\text { Length of Stay at Emergency Department in Hours } \\
\text { Mean (SD*) } \\
\text { Median (IQR**) } \\
\text { Minimum - Maximum } \\
\text { Not Reported \% (n) }\end{array}$ & $\begin{array}{l}9.37(10.37) \\
5(10) \\
0-52 \\
3.6(4)\end{array}$ \\
\hline $\begin{array}{l}\text { Length of Total Stay in the Hospital in Days } \\
\text { Mean }\left(\mathrm{SD}^{*}\right) \\
\text { Median }\left(\mathrm{IQR}^{* *}\right) \\
\text { Minimum - Maximum } \\
\text { Not Reported \% (n) }\end{array}$ & $\begin{array}{c}11.15(16.01) \\
6(8) \\
0.5-120 \\
4.5(5) \\
\end{array}$ \\
\hline $\begin{array}{l}\text { Mode of Transport \% (n) } \\
\text { Ambulance } \\
\text { Private Vehicle } \\
\text { Not Reported }\end{array}$ & $\begin{array}{c}7.2(8) \\
30.6(34) \\
62.2(69)\end{array}$ \\
\hline $\begin{array}{l}\text { Status at End of Hospitalization \% (n) } \\
\text { Discharged } \\
\text { Admitted to Ward } \\
\text { Dead } \\
\text { Not Reported }\end{array}$ & $\begin{aligned} & 8.1(9) \\
& 86.5(96) \\
& 4.5(5) \\
& 0.9(1)\end{aligned}$ \\
\hline $\begin{array}{l}\text { Referral from Ward to ICU \% (n) } \\
\text { Yes } \\
\text { No } \\
\text { Not Reported } \\
\end{array}$ & $\begin{array}{c}30.6(34) \\
60.4(67) \\
9.0(10)\end{array}$ \\
\hline $\begin{array}{l}\text { Type of Trauma \% (n) } \\
\text { Driver Involved Road Traffic Accident } \\
\text { Pedestrian Involved Road Traffic Accident } \\
\text { Fall Injury } \\
\text { Stab Wound } \\
\text { Burn } \\
\text { Drowning } \\
\text { Gun Shot Injury } \\
\text { Not Reported }\end{array}$ & $\begin{array}{c}28.8(32) \\
4.5(5) \\
26.1(29) \\
19.8(22) \\
7.2(8) \\
4.5(5) \\
1.8(2) \\
7.2(8)\end{array}$ \\
\hline $\begin{array}{l}\text { Blood Tests \% (n) } \\
\text { Done } \\
\text { Not Done } \\
\text { Not Reported }\end{array}$ & $\begin{array}{c}95.5(106) \\
4.5(5) \\
0.0(0)\end{array}$ \\
\hline $\begin{array}{l}\text { Assessment of Tetanus Vaccination Status \% (n) } \\
\text { Done } \\
\text { Not Done } \\
\text { Not Reported }\end{array}$ & $\begin{array}{c}6.3(7) \\
68.5(76) \\
25.2(28)\end{array}$ \\
\hline $\begin{array}{l}\text { Radiological Imaging \% (n) } \\
\text { Done }\end{array}$ & $87.4(97)$ \\
\hline
\end{tabular}




\begin{tabular}{|l|c|}
\hline Not Done & $9.9(11)$ \\
Not Reported & $2.7(3)$ \\
\hline Measurement of Heart Rate at Arrival \% (n) & $32.4(36)$ \\
Done & $67.6(75)$ \\
Not Done & $0.0(0)$ \\
Not Reported & $30.6(34)$ \\
\hline Measurement of Blood Pressure at Arrival \% (n) & $69.4(77)$ \\
Done & $0.0(0)$ \\
Not Done & \\
Not Reported & $30.6(34)$ \\
\hline Measurement of Oxygen Saturation Level at Arrival & $69.4(77)$ \\
\% (n) & $0.0(0)$ \\
Done & \\
Not Done & \\
Not Reported & $27.9(31)$ \\
\hline Measurement of Body Temperature at Arrival \% (n) & $72.1(80)$ \\
Done & $0.0(0)$ \\
Not Done & \\
Not Reported & $45.0(50)$ \\
\hline Number of Departments Involved in Management \% & $14.4(16)$ \\
(n) & $8.1(9)$ \\
One Department & $6.3(7)$ \\
Two Departments & $26.1(29)$ \\
Three Departments & \\
Four Departments & \\
Not Reported & $42.3(47)$ \\
\hline Type of Departments Involved in Management \% (n) & $2.7(3)$ \\
Surgical Departments Only & $7.2(8)$ \\
Orthopedic Department Only & $21.6(24)$ \\
Surgical and Orthopedic Departments & $26.1(29)$ \\
More than Two Departments & \\
Not Reported & \\
\hline Deviay, IQRe Inte-Quartle Range & \\
\hline
\end{tabular}

* $\mathrm{SD}=$ Standard Deviation; ** IQR= Inter-Quartile Range

Table 2: Characteristics of trauma patients admitted to King Abdulaziz University hospital stratified by length of total hospital stay $(\mathrm{n}=111)$

\begin{tabular}{|c|c|c|c|}
\hline Variable & $\begin{array}{l}\text { Length of Total } \\
\text { Hospital Stay } \\
\text { <6 Days }\end{array}$ & $\begin{array}{l}\text { Length of Total } \\
\text { Hospital Stay } \\
\geq 6 \text { Days }\end{array}$ & p-value \\
\hline $\begin{array}{l}\text { Age in Years } \\
\text { Mean }\left(\mathrm{SD}^{*}\right) \\
\text { Median (IQR**) } \\
\text { Minimum - Maximum }\end{array}$ & $\begin{array}{l}25.06(19.39) \\
20(30) \\
2-75\end{array}$ & $\begin{array}{l}26.22(16.69) \\
26(25) \\
1-69\end{array}$ & 0.75 \\
\hline $\begin{array}{l}\text { Gender \% (n) } \\
\text { Male } \\
\text { Female }\end{array}$ & $\begin{array}{c}51.9(41) \\
45.5 \%(10)\end{array}$ & $\begin{array}{l}48.1(38) \\
54.5(12)\end{array}$ & 0.59 \\
\hline $\begin{array}{l}\text { Nationality \% (n) } \\
\text { Saudi } \\
\text { Non-Saudi }\end{array}$ & $\begin{array}{l}53.5(23) \\
49.1(28) \\
\end{array}$ & $\begin{array}{l}46.5(20) \\
50.9(29) \\
\end{array}$ & 0.53 \\
\hline $\begin{array}{l}\text { Length of Stay at Emergency Department in Hours } \\
\text { Mean (SD*) } \\
\text { Median (IQR**) } \\
\text { Minimum - Maximum }\end{array}$ & $\begin{array}{c}10.44(10.20) \\
5.5(14) \\
1-44 \\
\end{array}$ & $\begin{array}{l}8.49(9.68) \\
5(7) \\
0-52 \\
\end{array}$ & 0.33 \\
\hline $\begin{array}{l}\text { Mode of Transport \% (n) } \\
\text { Ambulance } \\
\text { Private Vehicle }\end{array}$ & $\begin{array}{c}0.0(0) \\
46.7(14) \\
\end{array}$ & $\begin{array}{l}43.1(28) \\
53.3(16)\end{array}$ & 0.02 \\
\hline Status at End of Hospitalization \% (n) & & & \\
\hline
\end{tabular}




\begin{tabular}{|c|c|c|c|}
\hline $\begin{array}{l}\text { Discharged } \\
\text { Admitted to Ward } \\
\text { Dead }\end{array}$ & $\begin{array}{c}77.8(7) \\
46.6(41) \\
75.0(3)\end{array}$ & $\begin{array}{c}22.2(2) \\
53.4(47) \\
25.0(1)\end{array}$ & 0.16 \\
\hline $\begin{array}{l}\text { Referral from Ward to ICU \% (n) } \\
\text { Yes } \\
\text { No }\end{array}$ & $\begin{array}{c}16.7(5) \\
67.7(42)\end{array}$ & $\begin{array}{l}83.3(25) \\
32.3(20)\end{array}$ & $<0.001$ \\
\hline $\begin{array}{l}\text { Type of Trauma \% (n) } \\
\text { Driver Involved in Road Traffic Accident } \\
\text { Pedestrian Involved in Road Traffic Accident } \\
\text { Fall Injury } \\
\text { Stab Wound } \\
\text { Burn } \\
\text { Drowning } \\
\text { Gun Shot Injury }\end{array}$ & $\begin{array}{l}46.4(13) \\
20.0(1) \\
60.7(17) \\
60.0(12) \\
0.0(0) \\
60.0(3) \\
50.0(1)\end{array}$ & $\begin{array}{l}53.6(15) \\
80.0(4) \\
39.3(11) \\
40.0(8) \\
100.0(7) \\
40.0(2) \\
50.0(1)\end{array}$ & 0.08 \\
\hline $\begin{array}{l}\text { Blood Tests \% (n) } \\
\text { Done } \\
\text { Not Done }\end{array}$ & $\begin{array}{c}50.0(49) \\
50.0(2)\end{array}$ & $\begin{array}{c}50.0(49) \\
50.0(2)\end{array}$ & 0.69 \\
\hline $\begin{array}{l}\text { Assessment of Tetanus Vaccination Status \% (n) } \\
\text { Done } \\
\text { Not Done }\end{array}$ & $\begin{array}{r}57.1(4) \\
44.9(31) \\
\end{array}$ & $\begin{array}{c}42.9(3) \\
55.1(38) \\
\end{array}$ & 0.24 \\
\hline $\begin{array}{l}\text { Radiological Imaging \% (n) } \\
\text { Done } \\
\text { Not Done }\end{array}$ & $\begin{array}{c}47.8(43) \\
80.0(8)\end{array}$ & $\begin{array}{c}52.2(47) \\
20.0(2) \\
\end{array}$ & 0.12 \\
\hline $\begin{array}{l}\text { Measurement of Heart Rate at Arrival \% (n) } \\
\text { Done } \\
\text { Not Done }\end{array}$ & $\begin{array}{l}51.4(18) \\
49.3(33)\end{array}$ & $\begin{array}{l}48.6(17) \\
50.7(34)\end{array}$ & 0.84 \\
\hline $\begin{array}{l}\text { Measurement of Blood Pressure at Arrival \% (n) } \\
\text { Done } \\
\text { Not Done }\end{array}$ & $\begin{array}{l}57.6(19) \\
46.4(32)\end{array}$ & $\begin{array}{l}42.4(14) \\
53.6(37)\end{array}$ & 0.29 \\
\hline $\begin{array}{l}\text { Measurement of Oxygen Saturation Level at Arrival \% (n) } \\
\text { Done } \\
\text { Not Done }\end{array}$ & $\begin{array}{l}51.5(17) \\
49.3(34)\end{array}$ & $\begin{array}{l}48.5(16) \\
50.7(35)\end{array}$ & 0.82 \\
\hline $\begin{array}{l}\text { Measurement of Body Temperature at Arrival \% (n) } \\
\text { Done } \\
\text { Not Done }\end{array}$ & $\begin{array}{l}51.6(16) \\
49.3(35) \\
\end{array}$ & $\begin{array}{l}48.4(15) \\
50.7(36) \\
\end{array}$ & 0.83 \\
\hline $\begin{array}{l}\text { Number of Departments Involved in Management \% (n) } \\
\text { One Department } \\
\text { Two Departments } \\
\text { Three Departments } \\
\text { Four Departments }\end{array}$ & $\begin{array}{l}33.3(16) \\
62.5(10) \\
37.5(3) \\
00.0(0)\end{array}$ & $\begin{array}{l}66.7(32) \\
37.5(6) \\
62.5(5) \\
100.0(7)\end{array}$ & 0.03 \\
\hline $\begin{array}{l}\text { Type of Departments Involved in Management \% (n) } \\
\text { Surgical Departments Only } \\
\text { Orthopedic Department Only } \\
\text { Surgical and Orthopedic Departments } \\
\text { More than Two Departments }\end{array}$ & $\begin{array}{l}33.3(15) \\
33.3(1) \\
71.4(5) \\
33.3(8)\end{array}$ & $\begin{array}{l}66.7(30) \\
66.7(2) \\
28.6(2) \\
66.7(16)\end{array}$ & 0.35 \\
\hline
\end{tabular}

* SD= Standard Deviation; ** IQR= Inter-Quartile Range

Table 3: Characteristics of trauma patients admitted to King Abdulaziz University hospital stratified by type of trauma $(\mathrm{n}=111)$

\begin{tabular}{|l|c|c|c|}
\hline Variable & $\begin{array}{c}\text { Road Traffic } \\
\text { Accidents }\end{array}$ & $\begin{array}{c}\text { Non-Road } \\
\text { Traffic Accidents }\end{array}$ & P-value \\
\hline Age in Years & & & \\
Mean (SD*) & $27.81(0.17)$ & $27.09(0.19)$ & 0.85 \\
Median (IQR*) & $26.00(30.50)$ & $26.00(27.5)$ & \\
Minimum - Maximum & $6-69$ & $1-75$ & \\
\hline Sex \% (n) & $34.1(29)$ & $65.9(56)$ & 0.41 \\
Male & \\
\hline
\end{tabular}




\begin{tabular}{|c|c|c|c|}
\hline Female & $47.1(8)$ & $52.9(9)$ & \\
\hline $\begin{array}{l}\text { Nationality \% (n) } \\
\text { Saudi } \\
\text { Non-Saudi }\end{array}$ & $\begin{array}{l}59.5(25) \\
20.7(12)\end{array}$ & $\begin{array}{l}40.5(17) \\
79.3(46)\end{array}$ & 0.001 \\
\hline $\begin{array}{l}\text { Length of Stay at Emergency Department in Hours } \\
\text { Mean (SD*) } \\
\text { Median (IQR**) } \\
\text { Minimum - Maximum }\end{array}$ & $\begin{array}{l}11.27(0.12) \\
6.00(13.50) \\
1-48\end{array}$ & $\begin{array}{c}9.02(9.83) \\
5.00(10.00) \\
0.5-52\end{array}$ & 0.31 \\
\hline $\begin{array}{l}\text { Length of Total Hospital Stay in Days } \\
\text { Mean (SD*) } \\
\text { Median (IQR**) } \\
\text { Minimum - Maximum }\end{array}$ & $\begin{array}{l}12.25(13.01) \\
8.00(11.75) \\
0.5-55\end{array}$ & $\begin{array}{c}11.01(18.00) \\
5.00(8.00) \\
0.5-120\end{array}$ & 0.72 \\
\hline $\begin{array}{l}\text { Mode of Transport \% (n) } \\
\text { Ambulance } \\
\text { Private Vehicle }\end{array}$ & $\begin{array}{r}62.5(5) \\
37.5(12) \\
\end{array}$ & $\begin{array}{c}37.5(3) \\
62.5(20) \\
\end{array}$ & 0.23 \\
\hline $\begin{array}{l}\text { Status at End of Hospitalization \% (n) } \\
\text { Discharged } \\
\text { Admitted to Ward } \\
\text { Dead }\end{array}$ & $\begin{array}{c}12.5(1) \\
37.8(34) \\
40.0(2)\end{array}$ & $\begin{array}{l}87.5(7) \\
62.2(56) \\
60.0(3)\end{array}$ & 0.35 \\
\hline $\begin{array}{l}\text { Referral from Ward to ICU \% (n) } \\
\text { Yes } \\
\text { No }\end{array}$ & $\begin{array}{l}38.2(13) \\
37.1(23)\end{array}$ & $\begin{array}{l}61.8(21) \\
62.9(39) \\
\end{array}$ & 0.46 \\
\hline $\begin{array}{l}\text { Blood Tests \% (n) } \\
\text { Done } \\
\text { Not Done }\end{array}$ & $\begin{array}{c}36.0(37) \\
33.3(1) \\
\end{array}$ & $\begin{array}{c}64.0(64) \\
66.7(2) \\
\end{array}$ & 0.92 \\
\hline $\begin{array}{l}\text { Assessment of Tetanus Vaccination Status \% (n) } \\
\text { Done } \\
\text { Not Done }\end{array}$ & $\begin{array}{c}57.1(4) \\
44.9(31) \\
\end{array}$ & $\begin{array}{c}42.9(3) \\
55.1(38)\end{array}$ & 0.24 \\
\hline $\begin{array}{l}\text { Radiological Imaging \% (n) } \\
\text { Done } \\
\text { Not Done }\end{array}$ & $\begin{array}{c}38.7(36) \\
00.0(0)\end{array}$ & $\begin{array}{l}61.3(57) \\
100.0(8) \\
\end{array}$ & 0.08 \\
\hline $\begin{array}{l}\text { Measurement of Heart Rate at Arrival \% (n) } \\
\text { Done } \\
\text { Not Done }\end{array}$ & $\begin{array}{l}28.6(10) \\
39.7(27)\end{array}$ & $\begin{array}{l}71.4(25) \\
60.3(41)\end{array}$ & 0.27 \\
\hline $\begin{array}{l}\text { Measurement of Blood Pressure at Arrival \% (n) } \\
\text { Done } \\
\text { Not Done }\end{array}$ & $\begin{array}{l}36.4(12) \\
35.7(25)\end{array}$ & $\begin{array}{l}63.6(21) \\
64.3(45) \\
\end{array}$ & 0.95 \\
\hline $\begin{array}{l}\text { Measurement of Oxygen Saturation Level at Arrival \% (n) } \\
\text { Done } \\
\text { Not Done }\end{array}$ & $\begin{array}{l}30.3(10) \\
38.6(27)\end{array}$ & $\begin{array}{l}69.7(23) \\
61.4(43)\end{array}$ & 0.41 \\
\hline $\begin{array}{l}\text { Measurement of Body Temperature at Arrival \% (n) } \\
\text { Done } \\
\text { Not Done }\end{array}$ & $\begin{array}{l}40.0(12) \\
34.2(25)\end{array}$ & $\begin{array}{l}60.0(18) \\
65.8(48) \\
\end{array}$ & 0.58 \\
\hline $\begin{array}{l}\text { Number of Departments Involved in Management \% (n) } \\
\text { One Department } \\
\text { Two Departments } \\
\text { Three Departments } \\
\text { Four Departments }\end{array}$ & $\begin{array}{l}33.3(15) \\
40.0(6) \\
22.2(2) \\
85.7(6) \\
\end{array}$ & $\begin{array}{l}66.7(30) \\
60.0(9) \\
77.8(7) \\
14.3(1) \\
\end{array}$ & 0.04 \\
\hline $\begin{array}{l}\text { Type of Departments Involved in Management \% (n) } \\
\text { Surgical Departments Only } \\
\text { Orthopedic Department Only } \\
\text { Surgical and Orthopedic Departments } \\
\text { More than Two Departments }\end{array}$ & $\begin{array}{l}26.2(11) \\
33.3(1) \\
50.0(4) \\
56.5(13)\end{array}$ & $\begin{array}{l}73.8(31) \\
66.7(2) \\
50.0(4) \\
43.5(10)\end{array}$ & 0.13 \\
\hline
\end{tabular}

* $\mathrm{SD}=$ Standard Deviation; **IQR= Inter-Quartile Range 


\section{Discussion}

Our study used data from 111 trauma patients who received medical care at the KAUH during the 5-year study period. This low number of cases questions the accessibility of KAUH to trauma patients. We found that the majority of trauma patients were young males and this observation corresponds to the epidemiological pattern reported in other parts of Saudi Arabia. ${ }^{9-11}$ Further, RTAs were the most common type of trauma (33.3\%) and were followed by fall injuries $(26.1 \%)$. This observation is consistent with the incidence of injuries in Ontario, Canada, during the period 2009-2010, where RTAs were found to be the most common cause of trauma (39\%), followed by fall-related injuries $(38 \%))^{7,12}$

Data on the mode of transportation to the hospital and the pre-hospital care was not documented in more than $60 \%$ of the trauma patients implying major deficits in the documentation of trauma care. Unfortunately, KAUH didnot use a trauma sheet during the study period, which made documentation of trauma care unsystematic. Specifically, while our records show that in the majority of trauma patients radiological imaging (87.4\%) and basic blood tests $(95.5 \%)$ were conducted; toxicological screening and alcohol tests were never considered. Additionally, vital parameters such as heart rate, blood pressure, oxygen saturation, and body temperature were not universally assessed. Unfortunately, these practices are contrary to available evidence on trauma management. Specifically, the World Health Organization (WHO) recommendations consider alcohol as one of the major risk factors for the increase in RTA-related fatalities ${ }^{4}$ and Collins et al. have demonstrated that failure to adequately document trauma care is linked to higher mortality. ${ }^{13}$ Thus, at the KAUH, essential information such as toxicological profile and vitals needs to be recorded for all trauma patients immediately after arrival at the ER using proven and efficient systems such as internationally available trauma sheets. A majority of the cases with known pre-admission history arrived in private cars and less than one-fifth used ambulances. Previous studies have found that inadequate ambulance services for trauma patients were associated with higher mortality in low and middle income countries. ${ }^{12,14}$ However, due to the absence of data on trauma patient transport and the low rate of in-hospital mortality in our study, we could not assess such an association in our study population.

Many trauma patients presenting to our ER required admission with mean hospital-stay duration of $11.15 \pm 16.01$ days. Howard et al. have reported that the average length of hospital stay was 9.7 days among 80,544 trauma patients from 139 North American hospitals. ${ }^{15}$ This duration is slightly lower than our results and may reflect difference in severity or clinical practice. However, another study involving299 trauma patients has reported a much shorter length of hospital stay of 5.5 days. ${ }^{16}$ Fortunately, more than half of trauma patients presenting to our ER did not require ICU care; however, 2 patients died in the ER and 3 died during the first week after admission. However, 3 of the 34 patients died during their ICU stay, all within the first week of ICU admission. Additionally, 2 patients died during the emergency resuscitation within 2 hours of arrival. Similar results were found in a study on trimodal death distribution by Baker et al., where 3 out of 5 fatalities happened within 24 hours after admission. ${ }^{17}$

Longer length of hospital stay was significantly associated with transportation by ambulance, admission to the ICU, treatment by a greater number of specialties, and a tendency to be associated with non-RTAs. However, the association between length of hospital stay and mode of transport should be interpreted with great caution as the data on mode of transport was missing in $60 \%$ of the patients. Trauma due to causes other than RTAs were significantly more common among non-natives, were treated by a smaller number of departments, and showed a tendency to undergo radiological assessment more often. Surgical departments were most commonly involved in the care of trauma patients (42\%) and more than half of the patients $(55 \%)$ were treated by more than one department. These observations suggest that most patients suffered from poly-trauma. Payal and associates report similar results in a study that evaluated emergency management of poly-trauma in 210 patients from Northern India. They concluded that the mean number of consultations per patient was 3.2 and that the most commonly involved specialties were orthopedics (27\%) followed by neurosurgery $(22 \%) .{ }^{18}$

\section{Limitations}

To the best of our knowledge, this study is the first to describe trauma care at a Saudi teaching hospital in the western region of Saudi Arabia. Although KAUH is one of the major tertiary care and teaching medical centers in the western region of Saudi Arabia, we found considerable deficits in the volume and quality of trauma care provided. Although we included all trauma patients, and hence reduced the risk of patient selection bias, we could not reduce the risk of information bias due significant missing data. These, combined with the relatively small sample size are considerable limitations of our study. These limitations also precluded any analysis to estimate the strength of associations and our efforts to control for confounding factors using regression models.

\section{Conclusion}

Currently, trauma poses a significant threat to population health in Saudi Arabia and Jeddah in particular. Additionally, pre-hospital care should be improved in collaboration with relevant bodies such as ambulance services. On arrival at the ER, a comprehensive package of evidence-based investigations and treatments should be provided, documented, and regularly evaluated using internationally established trauma sheets. Further, KAUH should be upgraded to a trauma center that collaborates efficiently with other hospitals, ambulance services, and the police, utilizes evidence-based trauma guidelines for diagnosis and management, and provides high quality care and investment in preventing injuries to reduce mortality, morbidity, and, ultimately, improve population health. 


\section{Acknowledgments}

This project was funded by the Deanship of Scientific Research (DSR) at King Abdulaziz University, Jeddah, under grant no. J-742-140-38. The all authors therefore, acknowledge with thanks to the DSR for technical and financial support.

\section{Competing interests}

The authors declare that they have no competing interests.

\section{References}

1. Morbidity and Mortality Weekly Report. 2017:1-28

2. Injuries Violence the Facts. 2014:1-20

3. Alberdi F, Garcia I, Atutxa L, Zabarte M. Epidemiology of severe trauma. Medicina Intensiva (English Edition). 2014;38(9):580-8.

4. World Health Organization (WHO). Violence, Prevention I, World Health Organization. Global status report on road safety 2013: supporting a decade of action. World Health Organization; 2013;285-92.

5. ER. Panorama Special Traffic Issue 2013. 2013 Feb 9;:1-22.

6. Ansari S, Akhdar F, Mandoorah M, Moutaery K. Causes and effects of road traffic accidents in Saudi Arabia. Public Health. 2000;114(1):37-9.

7. Saudi Arabia SAMA. Saudi Arabian Monetary Agency. 2008;22;:1-417.

8. Alshahrani MS. Effect of private versus emergency medical systems transportation in motor vehicle accident victims: Trauma Center Experience in Saudi Arabia. J Family Community Med 2017;24(1):30-3.

9. Alghnam S, Alkelya M, Al-Bedah K, Al-Enazi S. Burden of traumatic injuries in Saudi Arabia: lessons from a major trauma registry in Riyadh, Saudi Arabia. Ann Saudi Med 2014;34(4):291-4.

10. Ansari S, Akhdar F, Mandoorah M, Moutaery K. Causes and effects of road traffic accidents in Saudi Arabia. Public Health 2000;114(1):37-9.
11. Mansuri FA, Al-Zalabani AH, Zalat MM, Qabshawi RI. Road safety and road traffic accidents in Saudi Arabia: a systematic review of existing evidence. Saudi Med J 2015;36(4):418-21.

12. Ontario Trauma Registry 2011 Report: Major Injury in Ontario, 2009-2010 Data. 2017 Sep 23:1-133

13. Collins SA, Cato K, Albers D, Scott K, Stetson PD, Bakken S, Vawdrey DK. Relationship between nursing documentation and patients' mortality. Am J Crit Care 2013;22(4):306-13.

14. Mock CN, Jurkovich GJ, Arreola-Risa C, Maier RV. Trauma mortality patterns in three nations at different economic levels: implications for global trauma system development. J Trauma Acute Care Surg 1998;44(5):804-14.

15. Husum H, Gilbert M, Wisborg T, Van Heng Y, Murad M. Rural prehospital trauma systems improve trauma outcome in low-income countries: a prospective study from North Iraq and Cambodia. J Trauma Acute Care Surg 2003;54(6):1188-96.

16. Champion HR, Copes WS, Sacco WJ, Lawnick MM, Keast SL, Frey CF. The major trauma outcome study: establishing national norms for trauma care. J Trauma Acute Care Surg 1990;30(11):1356-65.

17. Payal P, Sonu G, Anil GK, Prachi V. Management of polytrauma patients in emergency department: An experience of a tertiary care health institution of northern India. World $J$ Emerg Med 2013;4(1):15-9.

18. Padalino P, Intelisano A, Traversone A, Marini AM, Castellotti N, Spagnoli D, Russo R, Zola R, Salvini P. Analysis of quality in a first level trauma center in Milan, Italy. Annali Italiani $d i$ Chirurgia 2006;77(2):97-106.

How to cite this article: Yahya A, AlQulayti W, Almaghrabi S, Tallab M, Moniem MA, Nabeela AA. Trauma care at King Abdulaziz University hospital: A retrospective cohort study. $J$ Community Health Manag 2019;6(3):86-93. 